\title{
The Impact of Sequencing Human Genome on Genomic Food \& Medicine
}

\author{
Hameed Khan \\ Department of Genetics \& Robotics, NCMRR (National Center for Medical Rehabilitation Research), National Institutes of Health (NIH), \\ Bethesda, Maryland, USA
}

Email address:

Hameedkhan111@comcast.net

\section{To cite this article:}

Hameed Khan. The Impact of Sequencing Human Genome on Genomic Food \& Medicine. International Journal of Genetics and Genomics. Vol. 9, No. 1, 2021, pp. 6-19. doi: 10.11648/j.ijgg.20210901.12

Received: March 4, 2021; Accepted: March 30, 2021; Published: April 16, 2021

\begin{abstract}
This abstract describes the development of novel food and drug based on the genetic make-up individuals and their diseases. It also describes how sequencing of human genome help us design the most nutritious food for the burgeoning population of world. Regardless of race, religions or place of origin, sooner or later we all have to become vegetarians. Non-vegetarians eat meat because it contains, out of 20, eight essential amino acids in concentrated form. Genome sequencing have already identified all the essential amino acid Codons which could be spliced in the Rice, Corn or Wheat genomes. Genetic tools are now available to splice essential amino acids Codons into Rice Genome. Using the genetic tools, we have successfully spliced Beta Carotene gene into the Rice Genome to produce Golden Rice. Upon ingestion of the Golden Rice, Beta Carotene is converted to Vitamin A which prevents blindness in children. Based on the genetic make-up of the host cells, we will cut, paste and copy important genes to develop new food, new fuel, and new medicine to treat every disease known to mankind. Our future lies in Space. By 2024, we plan to land men on Mars. The inhabitants of Mars will grow genetically modified plants only. We will also discuss ethical issues based on the unintended consequences of genetic modification of our food and medicine.
\end{abstract}

Keywords: Nitrogen Mustard, Aziridine, Carbamate, Glioblastoma, Walker Carcinoma, AZQ

\section{Historical Background}

Genetic modification will provide the most nutritious food without meat. Regardless of race, religions or place of origin, sooner or later we all have to become vegetarians. The old definition of Vegetarianism is that it is a practice of abstaining from the consumption of all kinds of meat and meat products of slaughtered animals. The old definition must be replaced with new definition based on modern science. The origin of all living creatures such as animals, plants and humans on Earth is not a miracle. The appearance of life on Earth must be based on experimental evidence, reproducible and verifiable result. The old definition came from people's head too, but it was based on the information available to our elders of the Old-World Order. It was a time of Magical, Mystical, Devine intervention, Vital force or Spiritual process. Based on experimental evidence, now we know Life did not come from outer world, but it was evolved on Earth and Nature selects, we call this process the Natural Selection. We learned the complexity of Life, when we broke the genetic code and unlocked the secrets of life. When we brought the Genetic revolution, we entered a New-World Order based on the fact that the origin of life is based on the formation of the self-replicating RNA molecule. We need a new definition of vegetarianism such as Vegetarianism is a concept which provides food for human consumption enrich with all eight essential amino acids without meat.

This article examines the new Genomic Vegetarianism (producing edible vegetables based on our genetic makeup) and its impact after reading, mapping (sequencing) the book of life (Genome) of human and plants. We will make an attempt to explain how can we isolate, extract and transfer the essential ingredients (genes) from one species (animals) and transplant into another species (plants) to make most nutritious vegetarian food? We will also examine the ancient treatment of all diseases by plant products as is practiced in Ayurveda to modern Synthetic medicine based on the rational drug design to provide treatment based on the genetic makeup of a person.

Today, more than seven and a half billion people live on 
Planet Earth and we are adding 90 million new people each year. According to the UN estimate, by 2050, the population of the world is likely to reach nine billion. Do we have sufficient resources to feed, house and provide jobs to all the people? Or Do we want to see street riots, lawlessness, destructions of property and Chaos? We don't need dictatorship to control population explosion.

We have to think scientifically. The Old-World Order is gone. In primitive times when very little was understood about the nature of the living systems. The most routine biological activity such as germination of seed or the flowering of the plants were attributed to the Devine Intervention. It also appeals to those who felt debased by the implications that we are nothing more than a collection of molecules and our Free Will arose from the interaction of enormously large number of molecules.

Mystical Magical Age is over. The Age of Reason and Science has arrived. Science means knowledge. And how we get this knowledge? By conducting experiments. There is nothing debasing in the thought that we are made of atoms alone and thereby related to the rest of the Universe. Based on the scientific evidence, we have created a New World Order which refuse to believe in Devine intervention, Vitalism and the seven-day creation of life on Earth. The spontaneous generation of life is considered a myth and we rejects this belief in the absence of any evidence of the appearance of Life on Earth as a magical, mystical or spiritual process. We have New World Order which logically explains how the origin of life on Earth is by the formation of a self-replicating, self-evolving and self-organizing RNA molecule by an extremely slow process based on the interaction of basic elements found on Earth over billions of years.

This logical thinking is not a hypothesis. It is the New World Order which is based on the experimental evidence which provides reproducible and verifiable results. it was demonstrated in the Lab in the University of Chicago by Stanley Miller in 1953. To mimic the condition on early Earth, Miller took a round flask containing Nitrogen, Carbon dioxide, Water, the basic elements found on Earth, he sparked electric current, (to mimic thunder and lightning) and within a week, he converted the gases to dark oily substance containing amino acids leading to proteins which is the essential building blocks of life which perform all body functions.

Based on this information, we hypothesized that the appearance of life is based on the fact that a million-lightning strike Earth each day. At some remote corner of the Earth, lightning struck at a cloud of gases containing Nitrogen, Carbon dioxide, Water vapors, at a rock containing Phosphate whose interaction over three and a half billion years resulted in the creation of the first information molecules (RNA Ribonucleic Acid) called Nucleotides whose interactions give rise to a long string of letters called DNA (Deoxy Ribonucleic Acid) consisting of four nucleotides Adenine (A), Thiamine $(\mathrm{T})$, Guanine $(\mathrm{G})$ and Cytosine $(\mathrm{C})$. Nucleotide bases constituting DNA and RNA. All four nucleotides have also been synthesized in the Labs by Cyril Ponnamperuma of the University of Maryland and they all carry the same information and perform the same functions in all living creatures as natural DNA and RNA. The essence of life is information and the information is located on DNA. Once the RNA is formed, evolution started forming the double stranded DNA. It is the double stranded DNA, which is transcribed into a single stranded of RNA and which is translated in Ribosomes to amino acids. The interaction of amino acids give rise to proteins whose interaction give rise to first living cells on Earth.

From the first cell to a complete human being, the biochemical detail is so similar that is highly likely that all organism from plants to animals on Earth are evolved from a single cell. This hypothesis, that all living creatures have a common ancestor, is supported by a variety of observations. For example, all organisms are composed of one or more cells. The organization and function of these cells have enormous similarities in their biochemistry such as their photosynthetic and respiratory apparatus; in the detail of their reproductive behavior of the cells; in the ubiquity of the molecule DNA as the genetic material, passing information from the parent's cell to daughter cells; in the detail of the metabolic breakdown of the food extracting energy; and in the symmetry of the constituent's molecules. Even in the microscopic detail of the membrane structure including the molecular basis of the color of their skin. Using the same material and the same method, all living cells are evolved over and over in extremely diverse aggregate to forms plants and animals which we describe collectively as Life.

Millions of cells interact to give tissues which interact to give plants. About six hundred million years ago, it is the plants that brought Oxygen to Earth. The plants have the ability to conduct Photosynthesis that is in the presence of water, Carbon dioxide and Sunlight, the genes (a strip of DNA and the specific collection of nucleotides) of the Chloroplast in plants convert Carbon dioxide to its food Carbohydrates and in this process, it releases Oxygen as its by-product. Accumulation of Oxygen over millions of years accelerated Biological evolution leading to Natural Selection resulting in the appearance of Aerobics Life on Earth from microbe to plants to animals including humans.

We read the entire book of life (Genomes) of humans and many plant and animal species, letter by letter, word by word and sentence by sentence (sequencing) reading all essential ingredients (genes) in the book of their lives (Chromosomes). New generation of scientists are now sequencing the genomes of all living species on planet Earth. We have about three million known and 30 million unknown species. We have developed the tool kit of restriction enzymes (molecular scissors) to cut paste and copy and move around genes from one species to another. It is easier to grow plants than animals. We can make genetically rich plants which serve as factories to produce new food (by inserting essential amino acids codons), new fuel (using Matheno-coccus found at the bottom of the ocean floor which converts Carbon dioxide to Methane, a fuel. The ocean surface around the world is releasing enormous amount Methane. If it is not collected, it will harm the atmosphere as a Greenhouse gas responsible for causing 
Global Warming) and new medicine (by inserting antibiotic genes) to treat every disease known to mankind.

\section{The Impact of Sequencing Pant Genome on Vegetarian Diet}

Although there are many diverse and complex techniques involved in genetic engineering, its basic principles are reasonably simple. There are five major steps in the development of a genetically engineered crop. But for every step, it is very important to know the biochemical and physiological mechanisms of action, regulation of gene expression, and safety of the gene and the gene product to be utilized. Even before a genetically engineered crop is made available for commercial use, it has to pass through rigorous safety and risk assessment procedures.

The first step is the extraction of DNA from the organism known to have the trait of interest. The second step is gene sequencing and cloning, which will isolate the pure gene of interest from the entire extracted DNA, followed by mass-production of the cloned gene in a host cell such as Yeast. Once it is cloned, the gene of interest is designed and packaged so that it can be controlled and properly expressed once inside the host plant. The modified gene will then be mass-produced in a host cell in order to make thousands of copies. When the gene package is ready, it can then be introduced into the cells of the plant being modified through a process called Transformation. The most common methods used to introduce the gene package into plant cells include Biolistic transformation (using a gene gun) or Agrobacterium-mediated transformation using Ti (Tumor Induced) Plasmid. For example, in the Genome of Ti-Plasmid spliced into the codons of the essential amino acid, we can harvest in Yeast to make millions of clones. Once the inserted gene is stable, inherited, and expressed in subsequent generations, then the plant is considered a transgenic. Backcross breeding is the final step in the genetic engineering process, where the transgenic crop is crossed with a variety of generations that possesses important agronomic traits, and selected in order to obtain high quality plants that express the inserted gene or codon in a desired manner. The length of time in developing transgenic plant depends upon the gene, crop species, available resources, and regulatory approval. It may take 6-15 years before a new transgenic hybrid is ready for commercial release.

We describe below the ways of creating most nutritious vegetarian food without meat to feed the burgeoning population of the world. The new food would be non-toxic, non-allergenic, and most nutritious. Their nutritional content would be comparable to their non-vegetarian counterpart. We will examine how vegetables can also serve as factories for producing modern medicine and finally, as the food supplies increases, the population also increases. we will examine the Ethical problem of population explosion.

To provide meat to vast majority of non-vegetarians, we need to raise millions of live stocks. All our daily function is performed by our body using 20 amino acids in different combination making proteins. Deficiency in any one of those amino acids cause our health problem. Out of 20 amino acids, our body does not make eight amino acids. We call them essential amino acids and they are: Valine, Leucine, Isoleucine, Phenylalanine, Tryptophan, Lysine, Histidine, and Threonine. Although they are present in sufficient amount in the meat, but not in vegetables. We need outside source to introduce these amino acids in vegetables. They are present in some edible vegetables, but are present in very low quantities. We eat meat to receive all amino acids in sufficient amount.

Producing vast quantities of meat is detrimental to our health and the health of our planet. First, eating meat has its own problem. In over-cooked meat, the amino acids decompose to produce N-Oxide, a carcinogen. Second, to feed the world population, we have to raise millions upon millions of farm animals. In cattle's stomach grasses decompose to generate Methane, a Greenhouse gas responsible for impacting Climate Change. Instead of increasing the number of farm animals, we have to decrease animals to the lowest number, just enough to produce sufficient milk for the infants, and cheeses and Yogurts (curd) for adults. Instead of using animal milk, the adult population can now use various vegetable juices like the Almond Milk in their breakfast cereals. The fruit juices are not only good for our health, but also good for our environments. Fruit farms remove the excessive amount of Carbon dioxide from our atmosphere and reduce Greenhouse Effect.

In food producing animals, the cost of producing meat is very high. For example, to produce a pound of meat, we have to feed meat-producing animals about a hundred pound of edible vegetables. We cannot waste edible vegetables to feed animals. As predicted by UN, by 2050 , the World's population is likely to be nine billion. To feed nine billion people, how many more food producing animals do we have to raise? The answer is a lot.

Now we have a new solution to our food problem. Since we broke the genetic code and unlocked the secrets of life, we are ready to manipulate life not only to clean up our environment pollution, but also to create new food, new fuel and new medicine to treat every disease known to mankind.

After breaking the genetic code and unlocking the secrets of life, we are certain that through a process called Genetic Engineering, we could transfer the essential amino acids genes from the farm animals and insert into plants to enrich our vegetables diets with essential amino acids. The process of Genetic Engineering involves altering the genetic make of a plant using "Recombinant DNA Technology" which uses molecular scissors called the Restriction Enzymes to cut, paste and copy pieces of Codons which codes for the essential amino acids and transfer these codons from the animals to plants.

Before reading the plant genome, that is reading its entire book of life, it is essential to read the Human Genome. To read the entire Human Genome is a colossal undertaking requires billions of dollars and years of effort of thousands of scientists from around the world. To read the Human Genome not only 
requires the funding from multi-national governments, but also requires the effort of thousands of scientists from six industrialized nations and 20 biomedical centers. This effort is led by US followed by Germany, France, England, China and Japan. This was the greatest biological experiment ever conceived by Human mind. It will answer the most fundamental questions, we asked ourselves since the dawn of human civilization. What does it mean to be human? What is the nature of our memory and conscientiousness? And our development from a single cell to a complete human being? The biochemical basis of our senses and the process of our aging? The scientific basis of our similarity and dissimilarity? Similarities that all living creatures from the tiny blades of grass to the mighty Elephants including man, mouse. monkey and all plants from the plant kingdom are all made of the same chemical building blocks. And yet we are so diverse that no two individuals are alike. Even identical twins are not exactly identical, they grow up to become two separate individuals.

In 1990, US Congress authorized three billion dollars to our Labs in NIH (National Institutes of Health - an agency of US Government) to decipher the entire Human Genome to map, identify and locate all genes under the title, "The Human Genome Project." We found that our genome contains six billion four hundred million nucleotides bases half comes from our father and another half comes from our mother. Less than two percent of our Genome contains genes which code for proteins. The other 98 percent of our genome contains switches, promoters, terminators etc. The 46 Chromosomes present in each cell of our body are the greatest library of the Human Book of Life on planet Earth. The Chromosomes carry genes which are written in nucleotides. Before sequencing (determining the number and the order of the four nucleotides on a Chromosomes), it is essential to know how many genes are present on each Chromosome in our Genome. The Human Genome Project has identified not only the number of nucleotides on each Chromosome, but also the number of genes on each chromosome.

Our entire genome, the book of our life, is written in four nucleotides. As stated above, they are A, (Adenine) $\mathrm{T}$ (Thiamine), G (Guanine) and C (Cytosine). The chain of these nucleotides forms a double stranded string of nucleotides, one strand is inherited from our mother and another from our father, running in opposite directions called the DNA (Deoxy Ribonucleic Acid; a self- replicating, self-evolving and self-organizing molecule). According to Francis Crick's Central Dogma of Molecular Biology, [1] double stranded DNA is transcribed into a single stranded RNA (Ribonucleic Acid) which is translated in the Ribosome into proteins. The discovery of the double helical structure of DNA explained how the information to create life is stored, replicate, evolved and passed on to the next generation. This discovery opened a New World Order of ideas and buried the old explanation of the magical mystical appearance of life on Earth.

The double stranded DNA explained that the essence of life is information and the information is located on these four nucleotides. Every set of three nucleotide on the mRNA forms a codon which codes for a specific amino acid. The four-letter text of nucleotides forms a three letter Codon which codes for an amino acid. There are 64 different combinations of Codons which codes for all 20 amino acids. Sequencing human genome identifies the number of nucleotides and the order in which they are arranged. Less than two percent of our genome contains regulatory region, a piece of DNA, which controls the function of genes. More than 300 regulatory regions have been identified. More than ninety eight percent of our Genome contains non-coding region used to be called the Junk DNA which makes up to sixty percent of our entire Genome. The non-coding regions contains repetitive piece of DNA which is tightly packed and mostly remain silent. The sequencing of this region showed that the non-coding region is the part of Viruses and Bacteria picked up by our Genome during the millions of years of our evolutionary process. During Bacterial or Viral infection, the non-coding DNA could unfold transcribing into RNA resulting into hazardous protein which could create havoc for our health.

Gene, a strip of DNA, is the unit of inheritance. As I said above, out of four-letter text, that is A-T and G-C, and three letters code for an amino acid called the Codon. The starting Codon in a gene is the Codon AUG (instead of T nucleotide, we use $U$ nucleotide because Thiamin is converted to more water-soluble Uracil) which codes for amino acid Methionine. Long chain of DNA synthesis begins. The starting Codon is followed by a series of hundreds of Codons which codes for different amino acids in different species. There are three Stop Codons and they are AUG, UGG, and UGA. Once the stop codons appear, DNA synthesis stops. Bacteria and Viruses have short codon chain. The longest chain is in a gene of Ducharme Muscular Dystrophy, a neurological disease whose chain extends to two and a half million codons. Once a gene is identified, using Restriction Enzymes (molecular scissors) like EcoR1, we can cut, paste and copy all genes individually making a Restriction Site map. Once a single gene is isolated, we could compare the sequence of this gene with the Thousand Genome Project to identify differences called mutations which is responsible for causing diseases. Once mutated gene is identified, we can design drugs to shut off that gene. Sequencing Genome is like extracting Gold from its Ore.

\section{The Human Genome Sequencing}

The following list provide the details composition of human genome. It also provides the number of nucleotides and the number of genes present on each Chromosome [2-6].

We found that the Chromosome-1 is the largest Chromosome carrying 263 million A, T, G and C nucleotides bases and it has only 2,610 genes. The Chromosome-2 contains 255 million nucleotides bases and has only 1,748 genes. The Chromosome-3 contains 214 million nucleotide bases and carries 1,381 genes. The Chromosome- 4 contains 203 million nucleotide bases and carries 1,024 genes. The Chromosome-5 contains 194 million nucleotide bases and carries 1,190 genes. The Chromosome- 6 contains 183 million nucleotide bases and carries 1,394 genes. The Chromosome-7 
contains 171 million nucleotide bases and carries 1,378 genes. The Chromosome- 8 contains 155 million nucleotide bases and carries 927 genes. The Chromosome- 9 contains 145 million nucleotide bases and carries 1,076 genes. The Chromosome-10 contains 144 million nucleotide bases and carries 983 genes. The Chromosome-11 contains 144 million nucleotide bases and carries 1,692 genes. The Chromosome-12 contains 143 million nucleotide bases and carries 1,268 genes. The Chromosome-13 contains 114 million nucleotide bases and carries 496 genes. The Chromosome-14 contains 109 million nucleotide bases and carries 1,173 genes. The Chromosome-15 contains 106 million nucleotide bases and carries 906 genes. The Chromosome-16 contains 98 million nucleotide bases and carries 1,032 genes. The Chromosome-17 contains 92 million nucleotide bases and carries 1,394 genes. The Chromosome-18 contains 85 million nucleotide bases and carries 400 genes. The Chromosome-19 contains 67 million nucleotide bases and carries 1,592 genes. The Chromosome-20 contains 72 million nucleotide bases and carries 710 genes. The Chromosome-21 contains 50 million nucleotide bases and carries 337 genes. Chromosome-22 contains 56 million nucleotides and carries 701 genes. Finally, the sex chromosome of all female called the (X) contains 164 million nucleotide bases and carries 1,141 genes. The male sperm chromosome contains 59 million nucleotide bases and carries 255 genes.

If you add up all genes in the 23 pairs of Chromosomes, they come up to 26,808 genes and yet we keep on mentioning 24,000 genes needed to keep us function normally. Out of 24,000 gene, 16,000 are good genes which keep us healthy and 6.000 are mutated or bad genes which are responsible for causing six thousand different diseases and 2,000 pseudogenes which have lost their functions. As I said above, a gene codes for a protein, not all 24,000 genes code for proteins. It is estimated that less than 19,000 genes code for protein. Because of the alternative splicing, each gene codes for more than one protein. All functional genes in our body make less than 50,000 protein which interact in millions of different ways to give a single cell. Millions of cells interact to give a tissue, hundreds of tissues interact to give an organ and several organs interact to make a human.

The purpose of describing the sequence of the entire human genome is to map, identify and locate all genes and move any gene from animal genome to plant genome including edible vegetables to create a complete vegetarian's diet. Sequencing Human Genome also help develop new tool kit of enzymes, the molecular scissors, to cut, paste and copy any piece of DNA from one species and insert to another species including animal genes to plants genome. To feed the burgeoning population of the world, the Genetic Engineering tools will help us develop new food, new fuel and new medicine to treat every disease known to mankind.

\section{Genetically Modified Food}

To produce new vegetarian food, the genetic engineering procedures have successfully sequenced the Rice genome and have also enriched it producing Vitamin A and Iron rich Rice. Similar methods could also be used to introduce all eight essential amino acids in the cereals including Rice, Corn, Wheat genomes either by splicing a single codon to introduce a single amino acid or multiple codons to generate multiple amino acids to create most nutritious food for the burgeoning population of the world.

The following two methods are available to introduce novel genes in the plant genome: 1) The use of DNA particle gun for bombardment of an edible plant laced with the desired codon on either Tungsten or Gold pellet Gun, and 2) Transforming transgenic Agrobacterium tumefaciens-mediated codons for producing transgenic plant products carrying the essential amino acids Codons.

Using Particle Bombardment Method to introduce desired genes into plants:

Particle bombardment is a mechanical method of introducing the desired codon into plants.

The desired genetic sequence is cloned into a plant DNA Vector and introduced into the plant using the gene gun or particle gun. As in the common gun, the gene gun uses minute particles of Tungsten or Gold as the bullet. These particles are coated with the desired DNA solution and fired to the plant cells through the force of the Helium gas inside a vacuum-filled chamber. The DNA and the Tungsten/Gold particles get inside the cell, and within 12 hours, the inserted DNA gets inside the nucleus, integrate with the plant DNA and becomes functional. The Tungsten/Gold particles are sequestered to the vacuole and eliminated later.

\subsection{Using Agrobacterium Tumefaciens-Mediated Method to Introduce Desired Genes into Plants}

Transformed cells are cultured in vitro and introduced into small plants (regeneration) that express the inserted gene in Agrobacterium tumefaciens-mediated transformation

The "sharing" of DNA among living forms is well documented as a natural phenomenon. For thousands of years, farmers of livestock have been transferring different traits (genes) from one animal to another. For example, Agrobacterium tumefaciens, a soil bacterium known as 'nature's own genetic engineer', has the natural ability to genetically engineer plants. It causes Crown Gall disease in a wide range of broad-leaved plants, such as apple, pear, peach, cherry, almond, raspberry and roses. The disease gains its name from the large tumor-like swellings (galls) that typically occur at the crown of the plant, just above soil level. Ti-Plasmids (tumor induced plasmids) which serves as Vector (a carrier), are extracted from gall and used to make recombinant DNA. When harvested in Yeast, the Vectors make millions of clones (identical copies) for making novel food on large scale.

Basically, the bacterium transfers part of its DNA to the plant, and this DNA integrates into the plant's genome, causing the production of tumors like growth and associated changes in plant metabolism. Molecular biologists have utilized this biological mechanism to improve crops. The 
genes that cause the galls are removed and replaced with genes coding for desirable traits (essential amino acids Codons) and it retains its ability to replicate like gall. Plant cells infected with the bacterium will not form galls but produce cells containing the desired amino acids, which when cultured in a special medium will regenerate into plants and manifest the presence of essential amino acids. This will create the most nutritious vegetarian diet.

Either by Genetic transformation methods (Biolistic or Gene Gun or by Agrobacterium tumefaciens-mediated transformation methods), the main goal of genetic engineering in any transformation procedure is to introduce the gene of interest from human genome into the nucleus of the cell without affecting the cell's ability to survive. If the introduced gene is functional, and the gene product is synthesized, then the plant is said to be transformed. Once the inserted gene is stable, inherited and expressed in subsequent generations, then the plant is considered a transgenic.

Finally, Detection of Inserted Genes. Molecular detection methods have been developed to determine the integrity of the transgene (introduced gene or essential amino acid codons) into the plant cell. Sequencing of the whole genome of the transgenic plant should show the presence of newly introduced codon sequence.

Genomic food is based on the genetic make-up of the plant genome. For future space travelers, achieving Vegetarianism is the ultimate goal for humanity. As plant proteins are the primary sources of all dietary proteins consumed by human and animals and are inexpensive to produce in comparison with meat products; improving plant food quality will make a significant contribution to future needs of the population of the world. To provide most nutritious food to all of us, we have to cut, paste and copy codons of all essential amino acids in edible plants or seeds. There are more than 4,000 molecular scissors called Restriction Enzymes have been isolated from Bacteria. There are more than 3,500 multi-functional Type II Restriction Enzymes which are commercially available to cut, paste and copy fragments of essential amino acid codons either into double stranded DNA, or into a single stranded RNA of various lengths.

Fortunately, there are more than one codon which codes for the same amino acid. If one codon does not transfer easily from animal to plant, we could use the other codons. About a quarter million of flowering plants exist on Earth today. We cultivate just about 150 plants species for Agriculture purposes. To feed over seven billion people of the World, we cultivate a mere nine species of these plants on large scale. They are Corn, Rice, Wheat, Barley, Sorghum/Millet, Potatoes, Tomatoes, Sugar Cane and Soybean. The other vegetables, fruits and nuts are cultivated in smaller amounts. The Genomes of most of these edible plants have been sequenced. Luckily, there are only eight essential amino acids. It would be most useful to splice these codons in their genomes to produce the most nutritious food. The world's population will get all essential amino acids without eating meat or large quantities of vegetables. Besides fruits and vegetables, there are three major plants eaten by most people of the world and they need our immediate attention and they are Rice, Wheat and Corn.

Genes that carry essential amino acids are expressed in a two-step process and they are Transcription and Translation. First, the essential amino acids Codons are spliced or inserted into a double stranded of plant DNA which is later transcribed into a single stranded m-RNA. As I said above, it is the m-RNA which is translated in the Ribosomes into all 20 amino acids. The Cells decode m-RNA in groups of three nucleotides called Codons which carry instructions to produce the amino acids. As I said above, when double stranded DNA is transcribed into a single stranded m-RNA, the nucleotide Thiamin is converted to Uracil. The Methyl group of Thiamine is replaced by a more water- soluble Hydroxyl group forming the Uracil. The nucleotide $\mathrm{T}$ for Thiamin is replaced by $\mathrm{U}$ for the Uracil. The m-RNA is translated into amino acids in Ribosomes. The gene expression has a Start Codon (AUG) which codes for amino acid Methionine and there are three Stop Codon which are UGG, UAG and UGA. Once the Stop Codon appears at the tail end of the DNA, amino acids synthesis stops. The Codons for each essential amino acid and their alternative codons are described below:

Valine (GTT, GTC, GTA, GTG), Leucine (CTT, CTC, CTA, CTG; TTA, TTG), Isoleucine (ATT, ATC, ATA), Phenylalanine (TTT, TTC), Tryptophan (TGG), Lysine (AAA, AAG), arginine (CGT, CGC, CGA, CGG; AGA, AGG), Histidine (CAT, CAC), Methionine (ATG), Threonine (ACT, ACC, ACA, ACG).

Rice (Oryza sativa) is one of the most important crops in the world. Rice, Wheat, and Corn, together account for about half of the world's food production, and Rice itself is the principal food of half of the world's population. Using Genetic Engineering method, we must splice the essential amino acids codons in the Rice genome first. Rice contains 12 Chromosomes which carry 37,544 genes which are distributed over 400 to $430 \mathrm{Mb}$ nucleotides long DNA. Rice is consumed in most poor countries and more than two billion people around the world eat Rice. It is a good source of carbohydrate, proteins, fiber, lipid and fats, minerals (potassium, phosphorous, magnesium, calcium, sodium, copper and iodine) and vitamins (thiamine, riboflavin, niacin, vitamin B6 and folic acid). Unfortunately, Rice is devoid of essential amino acids in sufficient quantities. Biotech Rice with provitamin A (Golden Rice) has been developed and is being used to transfer Beta Carotene. Using the same Bio-tech methods, we can produce new food and new medicine in plant kingdom. For example, the transgenic Rice carries genes to produce Iron, Vitamin $\mathrm{A}$ and $\mathrm{E}$ and amino acid Lysine. We have also successfully spliced Bt genes (Bacillus thuringiensis) in the Rice Genome to introduce Bacterial resistant Rice against infectious worms.

Our next challenge is once we sequence the Rice genome, how many genes of essential amino acids, we could splice in a single Rice genome, one amino acid at a time or all eight in a single Rice genome. It depends upon the ease of insertion of a codon using a specific restriction enzyme. We have one major concern. It is known for centuries that when food supplies 
increase so does the population.

\subsection{Genetically Modified Fuel}

Each year, we collectively release 110 million tons of pollutants in our atmosphere. In addition, during 2017, the Methane emission alone reached 596 million metric tons primarily from agriculture - particularly from red meat and dairy producing animals and to a lesser extent from Rice farming. Although Methane is short-lived in the atmosphere than Carbon dioxide, but has a stronger Global Warming Effect. Of all the pollutants in the atmosphere, the major stable pollutant is Carbon dioxide. Carbon dioxide gas is responsible for causing Greenhouse Effect. Before the Industrial Revolution, the level of Carbon dioxide in our atmosphere was 175 PPM. After Industrial Revolution, the level of Carbon dioxide has gone up several fold. Today we have $400 \mathrm{ppm}$ Carbon dioxide in our atmosphere and at the current level, it is going up to 800 ppm by 2050. The main source of Carbon dioxide is from burning fossil fuel and from the decomposition of trees, volcanos and Oil refiners. There are more than a billion cars in the world today. Transportation alone produces $20 \%$ of the Carbon dioxide. Excessive Carbon dioxide is not only responsible for Global Warming, but also it destroys Coral Reef, Ice Caps and coast lines erosion causing sea level rise. To mitigate emission of this level, we need to develop a massive technological method to liquify Carbon dioxide to lock its emission and release at the ocean floor.

Marine cyanobacteria called Prochlorococcus thrive on the ocean floor. Their Chloroplast in the Chlorophyll Genome is responsible for Photosynthesis converting Carbon dioxide (a pollutant) to Oxygen. By conducting Photosynthesis on the ocean floor, these Cyanobacteria convert Carbon dioxide to their food Carbohydrate and produce Oxygen as a by-product. Fifty percent of the Photosynthesis is performed by these bacteria at the ocean floor and half of the Oxygen we breathe today comes from them. Photosynthesis is carried by billions of Microbes growing layer upon layer forming a thick carpet on the ocean floor near heat producing source like hydrothermal vent.

In the Gulf of California, the Pescadores Basin contains the deepest ocean floor. At a depth of 12,500 feet, it is known to have high-temperature hydrothermal vent in the Pacific Ocean. Dense colonies of Cyanobacteria, and tubeworms cling to rocks near the vent in high heat and noxious water. The chimneys on the ocean floor emits dark fluids that are rich in oil-like hydrocarbons and give off diesel-like smelly gases containing Methane, a fuel. Under deep dark ocean floor under tremendous pressure and extremely cold temperature a Cyanobacteria called Methano-coccus converts Carbon dioxide (a pollutant) to Methane (a Fuel). In our Lab, we could sequence the Genome of these bacteria brought from the ocean floor and cut, paste and copy their genes to produce large quantities of Methane in our Labs. Methane could be converted to Propane and Octane a better fuel.

\subsection{Genetically Modified Medicine}

We have also genetically engineered many vegetables including Potatoes which not only carries genes to enrich its starch content, but also to produce new medicine. We have produced edible vaccines in Corn, Banana and Potatoes. Now, we want to create a series of biological machines to produce new medicines to treat every disease known to mankind. Scientists at the Genentech were able to splice a human Insulin gene obtained from Human Pancreas and spliced into a microorganism to produce large scale of pure human Insulin to treat 300 million diabetics around the world. There are some important antibiotics which are used to treat most microbial infections and they are Neomycin, Kanamycin, Puromycin, Tobramycin, Apramycin, Amikacin, Gentamicin, Netilmicin etc. They are expensive because they are difficult to produce in large quantities. All antibiotics genes could be isolated and spliced in the plant genome to produce large quantities of inexpensive antibiotics to treat most microbial disease known to mankind. Could you imagine how much work lies ahead of us?

\subsection{Sequencing Ayurvedic Plant}

It is a common knowledge among the practitioners of Ayurveda that with the Ayurvedic treatment half of the patients respond to a certain drug and the other half do not. Now we know the reason, in humans, the genetic profile changes with aging. Our book of life that is our Genome is constantly changing. Genes sensitive to a certain Ayurvedic components are no longer active if the genetic profile changes.

Once the specific Ayurvedic component is identified for a specific genetic profile. The specific ingredient of the Ayurvedic plant is isolated. Large scale highly pure drug could be prepared using Polymerase Chain Reaction (PCR). The following ingredients are needed to produce large scale drug by PCR: First, we need a piece of double stranded DNA which codes for the active ingredient of the Ayurvedic medicine. Second, we need the four nucleotides (A/T and G/C). Third, we need 20-piece long DNA called forward and backward primers. Fourth, the most important enzyme the Taq-polymerase which is heat stable and is needed to complete the reaction. All we need is to heat the solution in water to 94 degree centigrade to break the Hydrogen bonds of double stranded DNA. As soon as the two single strands separate, the forward and backward primers join each strand, and the Taq-Polymer zip the nucleotides to produce two double stranded DNA of the drug. Simply heating cooling to 74 degree centigrade and adding the nucleotides, we can produce large quantity of the highly pure drug.

The length of time in developing transgenic plant depends upon the gene, crop species, available resources, and regulatory of approval. It may take 6-15 years before a new transgenic hybrid is ready for commercial release.

\subsection{Ayurvedic Medicine}

As a part of Ayurveda medicine at home, I was trained to extract useful drug from a plant called Rauwolfia Serpentina, locally known by different names such as the Indian snakeroot, devil pepper, or serpentine wood; it is a 
species of flower in the milkweed family. It is native to the Indian subcontinent and East Asia. Rauwolfia is a perennial undershrub widely distributed in the sub-Himalayan regions up to 1,000 meters. As a graduate student, I extracted the active ingredient Reserpine from the plant Rauwolfia Serpentina which is obtained from Patna, Bihar. Reserpine is used to treat hypertension in humans. Later, I worked on another plant called P. Harmala, extracting an antimicrobial alkaloid called Harmaline. Four alkaloids are separated from the seeds of P. harmala. Although Harmaline establishes the chief constituent, the other three were obtained in small quantities. One of the most active ingredients is identified as harmaline. Today, if I am asked to prepare large scale Reserpine, I would sequence Rauwolfia's genome and splice Reserpine gene in Yeast to produce large scale Reserpine.

\subsection{Genomic Medicine}

To continue my further studies, I was sent to England. At the London University, I was trained as an Organic Chemist in the Laboratory of Professor WCJ Ross of the Royal Cancer Hospital, a post-graduate medical center of the London University. I graduated from the University of London. After completing my doctorate and post-doctorate, I was offered a permanent job at the University of London.

Professor Ross was designing drugs to treat a variety of cancers such as Chlorambucil, Melphalan, Metroplan etc. [7-13] to shut off genes by attacking both strands of DNA simultaneously by cross-linking using Nitrogen Mustard analogs, which are extremely toxic.

As a part of my doctoral thesis, I was assigned a different path. Instead of cross-linking DNA, I am to design drugs to attack only one strand of DNA. This class of drugs is called Aziridines. Over the years, I made over 100 Dinitrophenyl Aziridines derivatives. One of them is Dinitro benzamide (CB1954) which gives a CI (Chemotherapeutic Index: ration of toxicity between normal cell to cancer cell) of 70 highest ever recorded. CB1954 wipes out a solid tumor by attacking the DNA of Walker Carcinoma 256, a solid aggressive tumor in Rat.

Nitrogen Mustards are highly toxic because they have neither specificity nor selectivity. They attack all dividing cells whether they are normal or abnormal. On the other hand, the analogs of Aziridines and Carbamates remain inactive in the basic and neutral media. They become activated only in the presence of Acidic media. In other words, Aziridines attack specifically acid producing cancer cells.

I used a simple rationale, the Aziridine attacks DNA in acidic medium, particularly the N-7 Guanine. The dye Dinitro benzamide has great affinity for Walker Tumor [14-16]. The Aziridine dinitro benzamide (CB1954) stain the tumor. As the tumor grows, it uses Glucose as a source of energy. Glucose is broken down to Pyruvic Acid. It is the acid which attacks the Aziridine ring. The ring opens to generate a Carbonium ion which attacks the most negatively charged N-7 Guanine of DNA shutting off the Walker Carcinoma gene in Rat.

To continue my work, I was honored with the Institute of
Cancer Research Post-Doctoral Fellowship Award of the Royal Cancer Hospital of London University. To increase the toxicity of CB1954 to Walker Carcinoma, I made additional 20 analogs as a postdoctoral fellow. When I attached one more Carbonium generating moiety, the Carbamate moiety, to the Aziridine Dinitrobenzene molecule, the compound Aziridine Dinitro benzamide Carbamate was so toxic that its Therapeutic Index could not be measured. We stop the work at the London University for the safety concern.

I continued my work on the highly toxic Aziridine/Carbamate combination in America when I was offered the Fogarty International Fellowship Award to continue my work at the National Cancer Institute (NCI) of the National Institutes of Health (NIH). I brought the idea from

London University of attacking one strand of DNA using not only Aziridine, but also Carbamate without using the same dye Dinitro benzamide $[17,18]$.

After working for about ten years at the London University, I moved to America when I was honored by the Fogarty International Fellowship Award by the National Institutes of Health, NIH, and the National Cancer Institute, NCI, of the USA. NIH has been my home for over a quarter of a century, I designed drugs to shut off mutated genes. All three Common Allele diseases have genetic origin. The rationale I used to synthesize anti-cancer drugs could be used to treat the other two old age diseases like Alzheimer or Cardiovascular Diseases.

In the following sections, I will describe in detail how anti-cancer drug like AZQ was designed to shut off Glioblastoma genes which cause Brain Cancer in humans. Using the same rational, we will consider how each of the other two diseases namely Cardiovascular Disease and Alzheimer could be treated by shutting off their genes to save human life: The order of these diseases is arranged based on the level of funding provided by NIH specifically by the NCI (National Cancer Institute).

My greatest challenge at NCI is to translate the animal work which I did in London University to humans. One day, I came across a paper which described that radio labeled Methylated Quinone cross the Blood Brain Barrier in mice. When injected in mice, the X-ray photograph showed that the entire radioactive Quinone was concentrated in the Mice's Brain within 24 hours. I immediately realized that Aziridine analogs of Quinone could be used to attack Glioblastoma multiforme, the brain tumor in humans, which is a solid aggressive tumor like Walker Carcinoma in Rats. I decided to use Quinone moiety as a carrier for Aziridine rings to attack Glioblastomas. Since Quinone has four substitution position available, by introducing an additional Carbamate moiety, I could increase its toxicity several folds. I planned to use this rational to translate animal work to human by introducing multiple Aziridine and Carbamate moieties to the Quinone to test against Glioblastomas in humans. Highest toxicity was obtained by attaching two Aziridines and two Carbamate moieties to Quinone, I named this novel compound AZQ. By treating brain cancer with AZQ, we observed that Glioblastoma tumor not only stop growing, but also start 
shrinking. I could take care of at least one form of deadliest old age cancers that is Glioblastomas. Literature search showed that AZQ is extensively studied.

As I said above, Glioblastomas, the brain cancers, is a solid and aggressive tumor and is caused by mutations on several Chromosomal DNA. Mutations on DNA is the result of damaging DNA nucleotides by exposure to radiations, chemical and environmental pollution, viral infections or genetic inheritance. The other factors responsible for causing DNA mutations are due to the fast rate of replication of DNA. For example, the bacteria E-coli grows so rapidly that within 24 hours, a single cell on a petri dish forms an entire colony of millions when incubated on the Agar Gel. Mistakes occur in DNA during rapidly replication such as Insertion of a piece of DNA, Deletion, Inversion, Multiple Copying, Homologous Recombination etc.

When an additional piece of nucleotide is attached to a DNA string, it is called Insertion or a piece of DNA is removed from the DNA string; it is called Deletion or structural Inversion of DNA is responsible for mutations. Since the gene in a DNA codes for Proteins, Insertion and Deletion on DNA have catastrophic effects on protein synthesis. Glioblastomas represent such an example.

All known Glioblastomas causing genes are located on five different Chromosomes and carries a total of 9,579 genes. It appears impossible to design drugs to treat Glioblastomas since we don't know which nucleotide on which gene and on which Chromosome is responsible for causing the disease. With the completion of 1,000 Human Genome Project, it becomes easier. By simply comparing the patient's Chromosomes with the Reference Sequence, the exact variants or mutations responsible for causing the disease could be identified.

Our next challenge is to identify in Glioblastoma which mutated nucleotides on which gene of which chromosome is attacked by AZQ. Radiolabeled AZQ provided the answer.

In Glioblastomas, three major changes occur on Chromosomes (C-7, C-9 \& C-10) and two minor changes occur on Chromosomes (C-1 \& C-19). These mutations are responsible for causing brain cancers in humans. In a normal human cell, Chromosome-7 which is made of 171 million nucleotide base pairs and it carries 1,378 genes. When Insertion occurs on Chromosome-7. Ninety-seven percent of Glioblastoma patients are affected by this mutation. On the other hand, a different mutation occurs on Chromosome-9 which is made of 145 million nucleotide base pairs and it carries 1,076 genes. A major Deletion of a piece of DNA occurs on Chromosome-9 which results in eighty- three percent patients who are affected by this mutation. A minor Deletion of DNA also occurs on Chromosome-10 which is made of 144 million base pairs and it carries 923 genes. Although it is a minor deletion of a piece of DNA and yet it contributes to ninety-one percent patients with Glioblastoma. To a lesser extent, small mutation occurs on Chromosome-1 (the largest Chromosome in our Genome). It is made of 263 million nucleotide base pairs and carries 2,610 genes) and Chromosome-19 (it is made of 67 million base pairs and carries 1,592 genes) is also implicated in some forms of Glioblastomas. Once the diagnosis is confirmed, the next step is how to treat the disease.

With the Quinone ring, I could introduce different combinations of Aziridine rings and Carbamate moieties and could create havoc for Glioblastomas. My major concern was how toxic this compound would be to the normal human brain cells. Fortunately, brain cells do not divide, only cancer cells divide.

Our Rational Drug Design using Aziridine/Carbamate work began in the University of London, England, and completed in the Laboratory of the National Cancer Institute (NCI), of the National Institutes of Health (NIH), in Bethesda, Maryland, USA. Over this period, we conducted over 500 experiments which resulted in 200 novel drugs. They were all tested against the experimental animal tumors. Forty-five of them were considered valuable enough to be patented by the US Government (US Patent 4,146,622). One of them is AZQ. Radiolabeled studies showed that AZQ has the ability to cross organ after organ, cross the Blood Brain Barrier, cross the nuclear membrane and attack the nuclear DNA shutting off the gene. X-ray studies showed that the radioactivity is concentrated in the tumor region. Glioblastoma stop growing and start shrinking [19].

A literature search shows that the International Scientific Community recognizes the significance of Dr. Khan's work. Using AZQ, they published more than 300 research papers in scientific literature. NIH considers his work is so valuable and innovative that he was honored with the "2004 NIH Scientific Achievement Award" one of the America's highest awards in Medicine. He is a Fellow of the American Institute of Chemistry and was elected to the American Science Advisory Board. He works at NIH, an agency of the U.S. Government.

He was also honored by the Government of India with the India's National Medal of Honor, "Vidya Ratna" a Gold Medal.

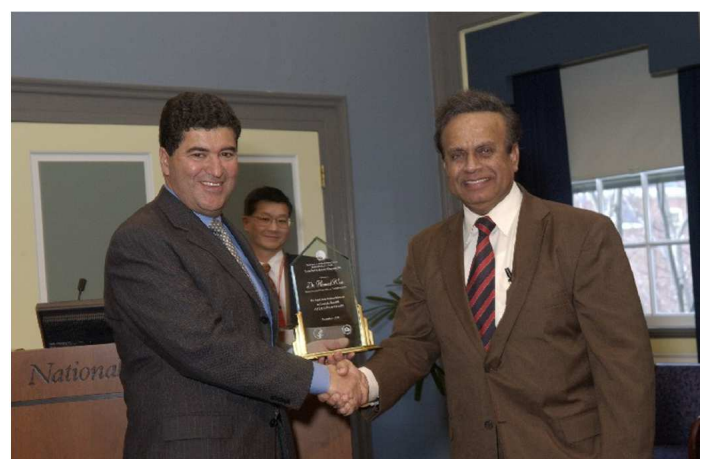

Figure 1. 2004 NIH Scientific Achievement Award. Presented to Dr. Hameed Khan By Dr. Elias Zerhouni, The Director of NIH During the NIH/APAO Award Ceremony held on December 3, 2004.

Dr. Khan is the Discoverer of AZQ (US Patent 4,146,622), a Novel Experimental Drug Specifically Designed to shut off a Gene that causes Brain Cancer for which he receives a 17-year Royalty for his invention (License Number L-0I9-0I/0). To this date, more than 300 research papers have been published on AZQ. The award ceremony was broadcast live worldwide by the Voice of America (VOA). Dr. Khan is the first Indian to receive one of America's highest awards in Medicine. 


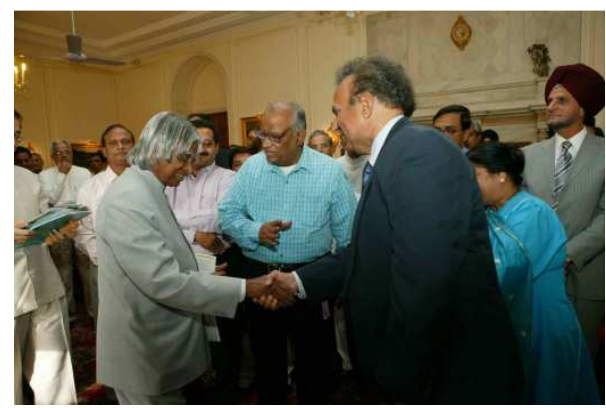

Figure 2. His Excellency, Dr. A. P. J. Abdul Kalam, The President of India Greeting Dr. A. Hameed Khan,

Discoverer of anti-cancer AZQ, after receiving 2004, Vaidya Ratna, The Gold Medal, One of India's Highest Awards in Medicine. At The Rashtrapathi Bhavan (Presidential Palace), in Delhi, India, During a Reception held on April 2, 2004
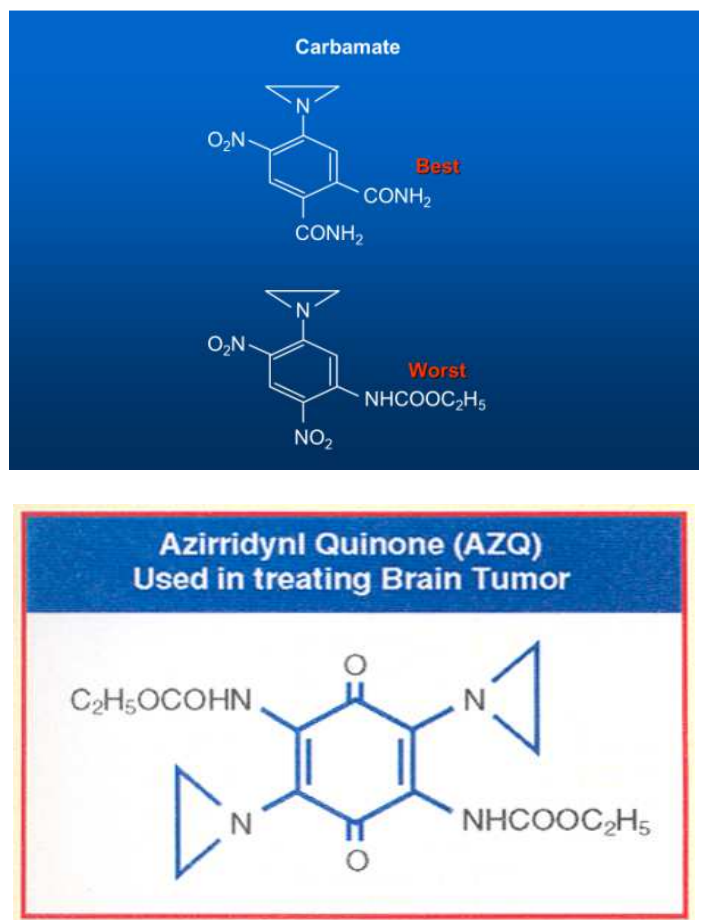

Figure 3. Single Strand DNA Binding Aziridine and Carbamate. (U.S. Patent 4,146,622).

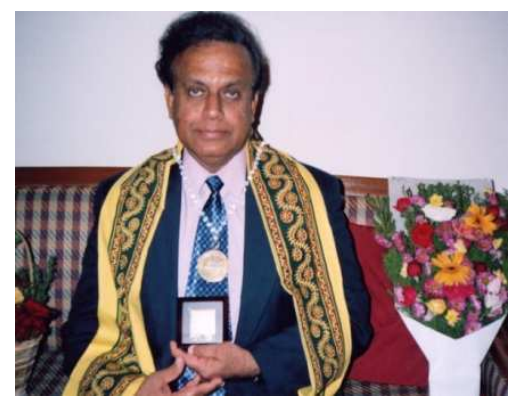

Figure 4. Gold Medal for Dr. Khan.

Dr. A. Hameed Khan, a Scientist at the National Institutes of Health $(\mathrm{NIH})$ USA, an American Scientist of Indian Origin was awarded on April 2, 2004. Vaidya Ratna; The gold Medal, one of India's Highest Awards in Medicine for his Discovery of AZQ (US Patent 4,146,622) which is now undergoing Clinical Trials for Treating Bran Cancer.

\section{Discussion}

Ethical Issues: (The Unintended Impact of Science on Society)

By producing new food, new fuel and new medicine, we ask ourselves what are the unintended consequences, for example, of over food production? Do we destroy excessive amount of food as the American farmers do each year or do, we control the world population? As the food supply increases, the population also increases. For example, at the time of India's Independence in 1947, the population of India was 400 million less than 75 years later, the population has increased to one Billion three hundred million.

By 2050, the population of the world will reach nine billion. By that time, we would have developed new food, new fuel, and new medicine to treat every disease known to mankind to protect, preserve and prolong human life beyond one hundred years, what would be the unintended consequences of Science on Society. This section discusses the unintended consequences of prolonging human life beyond one hundred years. The most nutritious vegetarian food containing all eight essential amino acids, will make world population lean healthy and long-lived. Besides new food, we would have new generations of plants which will serve as factories for producing new medicine based on the genetic make-up of the plants called the genomic medicine which will treat specific disease based on the specific individual genetic make-up. These class of medicines will further increase human lifespan.

Vegetarians live much longer than the non-vegetarians. We face the same population problem when we succeed in shutting off genes of all three old age diseases that is Cancer, Cardiovascular disease and Alzheimer. Most people on genetically engineered food will live longer and happier life. It raises several additional questions. What happens after we achieve that goal of reaching human lifespan to 100 years? What would be the quality of our life? By exercises and good nutrition, if the body mass is not retained, the Centenarians are most likely to be fragile and weak. They need the help of caretakers to perform the daily routine. By 2050, if we increase the age of about a hundred year of about a billion people, we need another billion caretakers. Will the society be happy with this achievement? I doubt it. The society is hardly likely to accept such a proposal.

As I said above, for deep space travel in search of habitable planets, we need to increase human lifespan beyond one hundred years. To cure diseases to prolong human life, several present and future attempts are described below:

We need to make two rationale approaches: First, to identify rare allele in the Genome of Centenarians responsible for prolonging their lives. Once identified the allele, we need to conduct genetic engineering that is to cut, paste, copy and splice and transfer the allele into the Genome of volunteers to study its function. Second, to design drugs to shut off genes of old age diseases to prolong life.

Next attempt to increase human life would be to prevent the loss of Telomeres, the six-letter code (TTAGGG) found at the tail end of each chromosome that shorten our DNA at each 
replication and shorten our lifespan. During replication, each Chromosome loses about 30 Telomeres each year. If we prevent the loss of Telomeres by using the enzyme Telomerase Reverse Transcriptase (TRT), we could slow down the aging process. We have already demonstrated in the worm C. Elegance that we could increase its lifespan several fold. Now, we could translate this work in human being; we could try by making a less virulent Flu Virus carrying TRT gene when injected to a volunteer who comes down with a mild Flu. When he recovers from the Flu, the TRT gene would have inserted in the entire genome of every cell in his body (we can confirm the insertion by sequencing his genome). Suppose at each replication only 15 Telomeres are deleted instead of 30 Telomeres. This person is likely to live twice as long. Also suppose the sequencing of his genome would confirms that we have successfully inserted in every cell of his body the TRT gene and also suppose that the longevity treatment with the Flu virus is safe, inexpensive and would be easily available to everyone, should we provide the treatment to every man, woman and child on the face of the Earth?

Such studies are likely to raise two serious ethical questions. First, we have to ask ourselves, do people have a right to live and second do we have a right to live as long as we wish, no matter how old, how weak or how sick we are? The answer to first question is, according to the UN charter, we all have the right to life, liberty and pursuit of happiness. It is the second question which is troublesome. Do people have a right to extent their lives as long as they wish? Most people are reluctant to answer this question either No or Yes. Both answers have some support.

Those who said No, have a good reason. First, they argue that there are seven and a half billion people live on planet Earth and we are adding 90 million additional people each year. As I said above, according to UN estimate, by 2050, the population of the world is likely to reach nine billion. Does our planet Earth have all resources to support such a population explosion? Our immediate problem is, can we provide food, fuel and medicine to all the people of the world? In poor countries millions of people are starving now. By extending lifespan, we will have serious problems such as lawlessness, riots and chaos in the streets. Moreover, the current population of Earth have polluted the water, polluted the air and polluted the land. Today, they wonder if the water they drink is safe, the food they eat is safe and the air they breathe is safe. If we continue to pollute the Planet with the current rate which is 110 million ton of pollutant that we release in the atmosphere each year; how much pollutants we would accumulate in the atmosphere in ten years or in hundred years?

On the other hand, those who say Yes; we should extend human lifespan have good reasons as well. We have no Plan B to save human life on some other Planet. We look up to Heaven to find another home for humanity. To search for a suitable Planet for human life to survive, we need to train an army of Astronauts to travel into deep space with extended life span. They may have to travel for centuries to find a habitable planet for humans. We do not want them to die on their way to find a new home for humanity. We must continue to search for treatment to prolong human life. Some simple-minded people, mostly religious people would say God created this wonderful Earth for us. Why can't we live on Earth forever? Since God has created us, He will protect us. Science say that we cannot stay on Earth forever. We have limited time on Earth. If we want intelligent human life to survive, we must leave Earth. For deep space travel, we need to increase our life span beyond one hundred years

In spite of the population explosion and environmental pollution, we must continue to work to extend human life beyond 100 years. The Universe is vast. Universe must be teeming with life, but the distances are so vast, with the current technological development, it is impossible to travel between star systems within the same galaxy. Traveling between galaxies is unthinkable at this time. There must be star systems which are formed earlier than our Solar System must have planets whose population must be technologically more advance than us. We must continue to develop technologically and must find a suitable home for humanity in another Solar System before our Sun dies.

According to the science of Cosmology, 13.7 billion years ago, the Universe was a single mass of energy. (May be God said let there be light and there was light) The Universe exploded with a Titanic force. Over billions of years, the cosmic dust cooled to form the islands of star systems called Galaxies. Each galaxy carries billions of stars. Our Sun is the only one star in our Solar System. The Solar System consists of $8 / 9$ Planets, 140 Moons and billions of comets and asteroids revolving around our Sun forming the Solar System. There are about 100 billion Solar Systems in our Milky Way Galaxy alone. There are over 400 Billion Galaxies in the visible part of the Universe. We have no idea how many more galaxies exist in the invisible part of the Universe.

Our Sun has been burning for the past four and a half billion years. It is a middle age dying star. It burns 700 hundred million tons of Hydrogen every second. It has used up more than half of its energy. Humanity is trapped in the middle age dying Solar System. We have a choice either to stay on Earth and die or to get out of this Solar System and survive. The Universe is a very big place. As I said above, the burning Sun has used up about half of its energy. Our Sun is mostly made of $74 \%$ of Hydrogen, $25 \%$ of Helium and one percent the other elements. Under intense temperature and pressure, Hydrogen atoms fused to form Helium and releases subatomic particle like Photons as Sunlight which travels 93 million miles (AU: Astronautical Unit) to reach Earth as light in eight minutes. As it continues to burn, the Helium is converted to Carbon. As more and more Hydrogen is used up, the Sun begins to cool and begins to expand. As it expands, the outer rim of the Sun begins to evaporate, melt and engulfs the nearest Planets Mercury and Venus within the Sun. On further cooling, the Sun will expand its outer rim even further approaching Earth. As its expansion reaches Earth, the intense temperature will boil off oceans, incinerate all life forms including us. As it exhausts its energy, the Sun will expand no further; it will collapse on itself and exploding as Super Nova. The explosion forms all other 120 elements from Iron to Gold including all essential element to make us. We are made of Star Dust. The 
Titanic explosion will destroy the entire Solar System. Before we are totally destroyed, we must leave this Solar System. Our responsibility is to protect, preserve and spread Human Intelligence in every corner of the Universe. On Religious ground, if we decide to stay and do nothing believing that God has created us, if we pray hard enough and long enough, God will protect us and save us from all dangers. On the other hand, we could say that we must do something like save ourselves. Humanity has come on a cross-road. We have a choice. One path leads to complete annihilation, total destruction, and extinction. The other path leads to escape this Solar System to survive elsewhere in the Universe.

Mother Nature has not been very kind to us. To develop technologically for deep space travel, she should have created us at least a billion years ago. We could have populated many Solar Systems in the Milky Way Galaxy. The great tragedy is that we came out of Africa recently when the Sun has used up half of its energy. The first half Chimp/half human, Lucy, the mother of us all, who walked out of Haggar Valley, Ethiopia, about three and a half million years ago. In spite of this delay, we still have enough energy in the Sun to get out of this Solar System only if we don't destroy ourselves by either going to Nuclear War or inviting Environmental collapse, or Meteorite impact followed by global forest fire or Tectonic plate shift resulting colossal Tsunami drowning life under ocean. We made more scientific discoveries during the last twenty-five years than the entire history of humanity on Earth. We have enough technology to take humanity out this Solar System. We have planned to take the following baby steps:

In 2024, we plan to send men on Mars. It is the first step in the right direction. It will technologically prepare us to survive on Mars under extremely cold condition on a tree-less water-less planet without Oxygen. Once we learn to survive on Mars, we could use Mars as a base to launch un-manned spacecrafts to distant Star Systems in search of habitable planets for humanity. For deep space travel, Vegetarianism will be the order of day. Carrying livestock on a long-distance space journey is unthinkable. The food we consume will be developed based upon the genetic make-up of a specific individual; we have to eat special Genomic food to keep us healthy and to prolong our life span for deep space travel.

For those inhabitants of Planet Earth who chose to stay to see the final end of life on Earth, they could do us a favor; they could stay behind to broadcast what they witness on Earth the final sunset, the end of life on Earth; the inhabitants will feel the intense heat, witness the worldwide burning of Oxygen, the boiling off the oceans, the massive forest fire. They could broadcast live the final end of the Earth for the space travelers who might be light years away from Earth on the way to Alpha Century Star system in search of habitable planets for humans. As our Sun used up most of its energy, it will expand no further. They could broadcast us how the Super Nova will collapse on itself and the Sun will explode with Titanic force. The gravity of the planets orbiting around Sun will collapse and all other planets and their moons will fall on each other causing further explosions destroying the entire Solar System. The inhabitants of distant galaxy such as Andromeda Galaxy, five million light year from Milky Way, will see the destruction of Solar System as a tiny ripple on the third arm of the Milky Way Galaxy.

This lecture addresses to the space age vegetarians who wish to explore the Universe. We began our journey on Earth as Vegetarians and we will have to end our journey as Vegetarians as we plan to leave Earth. We have a long journey ahead of us. Frank Drake of SETI (Search of Extra-terrestrial Intelligence Institute) is waiting to receive radio-signals for the past 70 years, and have not received any reproducible signal so far. It may be that there is no technologically developed society within the 70 light year distance to respond to his radio-signals. Moreover, we have been sending Radio and TV signals for the past 100 years in every direction. We have not received any reply. It tells us that there is no technologically developed intelligent life on any Star System within a hundred Light Years. It also tells us how rare and precious is life in the Cosmos and that we must prepare for a long journey beyond 100 Light Year. We must learn to survive for centuries in our spacecrafts. We must learn to grow food, produce Oxygen and recycle water for the endless journey. Comets are water world. We must learn to capture comets and attach them to our space craft as a constant source of water and Oxygen for a long journey. To succeed and survive on an endless journey in space, we need outstanding men and women. Who among you will be the vanguard of research and technology to protect, preserve and spread human intelligence in every corner of the Universe? We bequeath the future of humanity in your hands; we know that you will do your best to take humanity out of this Solar System to a safer place in the Cosmos before the Sun dies.

\section{Conclusion}

What are the immediate problems back on Earth: What if we succeed in prolonging Human Life on Earth? What if we succeed tomorrow in developing treatment of all three old age diseases to double or triple the Human life for the deep space travelers? If we don't succeed tomorrow may be day after tomorrow. Say the treatment is safe, inexpensive and easily available to every man, woman and child on the face of the Earth. We face new Ethical problems. Who decides that person A will receive the longevity treatment and will live for a long-distance travel in space and person B who stays behind will not receive the same treatment and therefore will die? We need debate and discussion and come up with guidelines for our society. One person cannot provide answer to all these questions. All I want to do is to raise these questions in your mind. My aim will be fulfilled if I have made you think along these lines.

\section{Appendix}

\section{Test questions}

1. Did Life originate on Earth? Based on the scientific evidence, we have created a New World Order which refuse to believe in Devine intervention, Vitalism and the seven-day creation of life on Earth. The spontaneous 
generation of life is considered a myth and we reject this belief in the absence of any evidence of the appearance of Life on Earth as a magical, mystical or spiritual process. We have New World Order which logically explains how the origin of life on Earth is by the formation of a self-replicating, self-evolving and self-organizing RNA molecule by an extremely slow process based on the interaction of basic elements found on Earth over billions of years.

2. What is the New World Order? When we broke the genetic code and unlocked the secret of life and brought the Genetic revolution, we entered a New-World Order based on the fact that the origin of life is based on the formation of the self-replicating, self-evolving, and self-organizing RNA molecule, which has the ability to store information like DNA and catalyzed reaction like proteins.

3. Where the Oxygen came from on the early Earth? About six hundred million years ago, it is the plants that brought Oxygen to Earth. The plants have the ability to conduct Photosynthesis that is in the presence of water, Carbon dioxide and Sunlight, the genes (a strip of DNA and the specific collection of nucleotides) of the Chloroplast in plants convert Carbon dioxide to its food Carbohydrates and in this process, it releases Oxygen as its by-product. Accumulation of Oxygen over millions of years accelerated Biological evolution leading to Natural Selection resulting in the appearance of Aerobics Life on Earth from microbe to plants to animals including humans.

4. Why we read the Human Book of Life under the Human Genome Project? Our Genome carries 24,000 genes. Sixteen thousand good genes, six thousand bad genes and two thousand pseudo genes. Sequencing genome is like extracting Gold from its Ore; it is to identify the bad genes responsible for causing diseases, to cure diseases to design drugs to shut off those bad genes.

5. What are the Essential Amino Acids and why we need them? Proteins in our body is made of twenty amino acid. Our body makes all amino acids except the following eight and they are: Valine, Leucine, Isoleucine, Phenylalanine, Tryptophan, Lysine, Histidine, and Threonine. We need outside source like meat. Without the essential amino acids, we develop a variety of diseases.

6. Can we get essential amino acids in our diet without eating meat? Essential amino acids Codons are identified. We can insert these codons in the genomes of Rice, Wheat or Corn to produce the most nutritious food. The Codons for each essential amino acid and their alternative codons are described below: Valine (GTT, GTC, GTA, GTG), Leucine (CTT, CTC, CTA, CTG; TTA, TTG), Isoleucine (ATT, ATC, ATA), Phenylalanine (TTT, TTC), Tryptophan (TGG), Lysine (AAA, AAG), arginine (CGT, CGC, CGA, CGG; AGA, AGG), Histidine (CAT, CAC), Methionine (ATG), Threonine (ACT, ACC, ACA, ACG).

7. Scientists at the Genentech were able to splice a human Insulin gene obtained from Human Pancreas and spliced into a microorganism to produce large scale of pure human Insulin to treat 300 million diabetics around the world. What other important antibiotics which are used to treat most microbial infections could be spliced in the plant genome? Some of the important antibiotics are Neomycin, Kanamycin, Puromycin, Tobramycin, Apramycin, Amikacin, Gentamicin, Netilmicin etc.

8. How Genomic Medicine is different from Reactive medicine? Genomic medicine is different from the Reactive Medicine. If a Physician notice a high blood pressure in a patient, he lowers the blood pressure in the patient by treating with Plavix. Genomic medicine is a predictive medicine. Looking at the genetic make-up and the MRI of the patient, the Physician predicts the onset of a disease. We have to design drugs like AZQ to treat the disease before it becomes serious and untreatable.

\section{References}

[1] Watson, J. D., \& Crick, F. H. C. A structure for deoxyribose nucleic acid. Nature 171, 737-738 (1953).

[2] Genome Sequencing, Nature, 409,934-941, 2001.

[3] Genetics, Nature, 409, 660-921, 2001.

[4] Genomics, Nature, 431, 931-945, 2004.

[5] Genome Sequencing, 438, 803-810, 2005.

[6] Genomics, Nature, 550, 345-353, 2017.

[7] Chlorambucil - CancerConnect News". CancerConnect News. Retrieved 2015-12-21.

[8] Ross. W. C. J, "The Chemistry of Cytotoxic Alkylating Agents" In Advances in Cancer Research by Greenstein, J. P., and Haddow, A., Academic Press, Inc., New York, 397-449, (1953).

[9] Ross, W. C. J., "Biological Alkylating Agents" Butterworth, London, 1962.

[10] Ross, W. C. J., Journal of Chemical Society, 183, 1949.

[11] Biological Alkylating Agents, Ross, W. C. J., J. Chem. Soc., 2257 (1950).

[12] Ross, W. C. J., Mitchley, B. C. V., Ann. Rep. Brit. Empire Cancer Campn, 42, 70 (1964).

[13] Melphalan Lancet 370 (9594): 1209-18.

[14] L. M. Cobb, T. A. Connors, L. A. Elson, A. H. Khan, B. C. V. Mitchley, W. C. J. Ross and M. E. Whisson, BIOCHEMICAL PHARMACOLOGY, col. 18, pp. 1519-1527 (1969) "2,4-Dinitro-5-Ethyleneiminobenzamide (CB 1954): A Potent and Selective Inhibitor of the Growth of the Walker Carcinoma 256".

[15] A. H. Khan and W. C. J. Ross, CHEM.-BIOL INTERACTIONS, vol 1, pp. 27-47 (1969/70) "Tumour-Growth Inhibitory Nitrophenylaziridines and related compounds: Structure-Activity Relationships" PART I.

[16] A. H. Khan and W. C. J. Ross, CHEM.-BIOL INTERACTIONS, vol 4, pp. 11-22 (1971/72) "Tumour-Growth Inhibitory Nitrophenylaziridines and related compounds: Structure-Activity Relationships" PART II. 
[17] A. Hameed Khan and John Driscoll, JOURNAL OF MEDICINAL CHEMISTRY, vol. 19, No. 2, pp. 313-317 (1976) "Potential Central Nervous System Antitumor Agents: Aziridinylbenzoquinones. PART I.

[18] Ed Chou, A. Hameed Khan and John Driscoll, JOURNAL OF MEDICINAL CHEMISTRY, vol. 19, pp. 1302 (1976) "Potential Central Nervous System Antitumor Agents: Aziridinylbenzoquinones. PART II.

[19] "Aziridinyl Quinone: Anti-transplanted Tumor Agents". UNITES STATES PATENT \# 4,146,622, (March 27, 1979) Investors: John S. Driscoll; A. Hameed Khan; Feng-e-Chou, $\mathrm{NIH}$, Maryland, USA Additional Information is available at Facebook.com/hameed.khan 7773 .

\section{Biography}

Hameed Khan was born in Hyderabad, India, and educated in England. He received his Doctorate Degree in Chemistry from the University of London. As a Fogarty International Scholar Awardees, he came to America in the laboratories of the National Cancer Institute (NCI) of the NIH (National Institutes of Health). He has worked for the NIH for the past 25 years. Currently, he served as the Senior Scientist in NCMRR. Dr. Khan is the discoverer of (AZQ), Aziridine Quinone, which is a novel class of drug that passes across the Blood Brain Barrier and attack brain tumor DNA shutting off gene that causes Brain Cancer. 